\section{RMD Open}

Rheumatic \&

Musculoskeletal Diseases

\title{
Evaluation of the joint distribution at disease presentation of patients with rheumatoid arthritis: a large study across continents
}

\author{
Sytske Anne Bergstra, ${ }^{1}$ Arvind Chopra, ${ }^{2}$ Manjit Saluja, ${ }^{2}$ David Vega-Morales, ${ }^{3}$ \\ Nimmisha Govind, ${ }^{4}$ Tom W J Huizinga, ${ }^{1}$ Annette van der Helm-van Mil ${ }^{1}$
}

To cite: Bergstra SA, Chopra A, Saluja M, et al. Evaluation of the joint distribution at disease presentation of patients with rheumatoid arthritis: a large study across continents. RMD Open 2017;3:e000568. doi:10.1136/ rmdopen-2017-000568

- Prepublication history and additional material for this paper are available online. To view these files, please visit the journal online (http://dx.doi. org/10.1136/rmdopen-2017000568).

Received 1 September 2017 Revised 20 November 2017 Accepted 26 November 2017

\section{(a) CrossMark}

${ }^{1}$ Department of Rheumatology, Leiden University Medical Center, Leiden, The Netherlands 2Department of Rheumatology, Center for Rheumatic Diseases, Pune, India

${ }^{3}$ Department of Rheumatology, Universidad Autonoma de Nuevo Leon, San Nicolas de los Garza, Nuevo Leon, Mexico

${ }^{4}$ Department of Rheumatology, University of the Witwatersrand, Johannesburg, South Africa

Correspondence to Sytske Anne Bergstra; s.a.bergstra@lumc.nl

\section{ABSTRACT}

Background Genetic and environmental risk factors for rheumatoid arthritis (RA) are population dependent and may affect disease expression. Therefore, we studied tender and swollen joint involvement in patients newly diagnosed with RA in four countries and performed a subanalysis within countries to assess whether the influence of autoantibody positivity affected disease expression.

Methods Patients with symptom duration $<2$ years fulfilling the American College of Rheumatology/European League Against Rheumatism 2010 RA classification criteria were selected from METEOR (Measurement of Efficacy of Treatment in the Era of Outcome in Rheumatology), an international observational database, and the Dutch Leiden Early Arthritis Clinic. Indian ( $n=947)$, Mexican $(n=141)$, South African $(n=164)$ and Dutch $(n=947)$ autoantibodypositive and negative patients with RA, matched by symptom duration, were studied for swollen and tender joint distribution.

Results Between countries, the reported distribution of swollen joint distribution differed, with more knee synovitis in Mexico, South Africa and India compared with the Netherlands (37\%, 36\%, 30\% and 13\%) and more elbow $(29 \%, 23 \%, 7 \%, 7 \%)$ and shoulder synovitis $(21 \%, 11 \%$, $0 \%, 1 \%)$ in Mexico and South Africa compared with India and the Netherlands. Since the number of autoantibodynegative patients in Mexico and South Africa was limited, Indian and Dutch autoantibody-positive and negative patients with RA were compared. The number of swollen and tender joints was higher in autoantibody-negative patients, but the overall distribution of involved joints was similar.

Conclusion Joint involvement at diagnosis does not differ between autoantibody-positive and negative patients with RA in India and the Netherlands. However, joint involvement is reported differently across countries. More research is needed whether these differences are cultural and/or pathogenetic.

\section{INTRODUCTION}

The disease phenotype of rheumatoid arthritis (RA) may be influenced by different factors, including the presence of autoantibodies.

\section{Key messages}

What is already known about this subject?

- Studies describing the phenotype of rheumatoid arthritis (RA) in non-Western populations are rare but the available evidence suggests differences in RA phenotype in various populations.

What does this study add?

- This is the first large study comparing joint involvement in patients with recent-onset RA from different populations.

- In the Netherlands and India, the distribution of involved joints did not differ for autoantibodypositive and negative patients.

- Synovitis in large joints was more frequent in India (knees), South Africa (knees, elbows) and Mexico (knees, elbows, shoulders) than in the Netherlands.

How might this impact on clinical practice?

- The present findings are of interest for etiopathological studies.

Many patients with RA are positive to one or more autoantibodies, which can precede symptom onset by years. ${ }^{2}$ Currently, the two most important autoantibodies involved in the diagnosis and prognosis of RA are rheumatoid factor and anti-citrullinated protein antibodies (ACPA). ${ }^{3}$ Although these autoantibodies are thought to be involved in the disease pathogenesis, a proportion of patients with RA test negative for both, indicating that the presence of rheumatoid factor and/or ACPA is not a prerequisite for the development of RA. ${ }^{4}$ It is even suggested that ACPA-positive and negative RA might not be the same disease, supported by differences in genetic backgrounds. ${ }^{56}$

A previous study investigated differences in phenotype of ACPA-positive and negative RA in Dutch patients and found similar 
phenotypes at the time of diagnosis. ${ }^{7}$ However, the pathogenesis of RA is complex; genetic and environmental risk factors are involved and both are population dependent. In addition, local cells, systemic factors as well as local mechanical factors are suggested to influence site-specific inflammation. ${ }^{8-11}$ Environmental factors are inherently different in different parts of the world. The genetic make-up differs across the world and, consequently, different genetic risk factors for RA are identified in different populations. ${ }^{12}$

Nevertheless, most scientific research on RA is done in Western countries and in line with this, studies describing the phenotype of RA in non-Western populations are rare. Although patients were generally not evaluated at the time of diagnosis, the scarcity of available evidence suggests differences in RA phenotype in various populations. ${ }^{1314}$ Therefore, we studied the distribution of joint inflammation at the time of diagnosis in different RA populations (Mexican, Dutch, Indian and South African). In addition, within the Indian and Dutch populations, the joint distribution was subsequently compared between autoantibody-positive and negative patients with RA.

\section{METHODS}

\section{Populations}

Patients fulfilling the American College of Rheumatology/European League Against Rheumatism 2010 RA classification criteria ${ }^{15}$ with available joint counts and symptom duration $<2$ years at the time of diagnosis were selected from two observational databases. Dutch patients were selected from the Leiden Early Arthritis Clinic (EAC) cohort. This is an inception cohort including patients presenting with symptoms $<2$ years and clinically confirmed arthritis at the Leiden University Medical Center (LUMC), which is the only centre for rheumatic diseases in a semirural area with $>400.000$ inhabitants. Indian, Mexican and South African patients diagnosed with RA were selected from Measurement of Efficacy of Treatment in the Era of Outcome in Rheumatology (METEOR). This is a large international, observational database including patients with a diagnosis of RA according to the rheumatologist, capturing daily clinical practice. Indian patients were included in a private rheumatology facility in a community setting (Pune). South African patients were included in a large provincial hospital (Johannesburg). Mexican patients were selected from two university hospitals and one regional hospital (Monterrey and Mexico City). In all patients included in this study, the 2010 classification criteria were only applied to patients with a clinical suspicion or diagnosis of RA according to the rheumatologist and patients who fulfilled these criteria were studied. All antibody measurements were performed locally. Both the EAC and the METEOR databases have been described extensively before. ${ }^{16} 17$ The EAC was approved by the Medical Ethics Committee of the LUMC and all participants gave written informed consent. Data in the METEOR database were gathered anonymously and captured only daily clinical practice; here informed consent was not required.

\section{Joint counts}

The main outcome was the swollen joint distribution at time of diagnosis. Tender joint distribution was also studied. Forty-four swollen and 53 tender joint counts were collected in Dutch and Indian patients; 28 swollen and tender joint counts in Mexican and South African patients. Small joints included metacarpophalangeal (MCP), proximal interphalangeal (PIP), metatarsophalangeal (MTP) 2-5, thumb, interphalangeal and wrist joints. Large joints included shoulders, elbows, hips, knees and ankles, similar to the definitions used in the 2010 criteria. $^{15}$

\section{Sensitivity analyses}

Because joint distribution is part of the 2010 classification criteria, hence generating circularity between this inclusion criterion and the outcome of interest, joint distributions were also studied for patients diagnosed with RA by the treating rheumatologists (hence ignoring classification criteria).

\section{Statistics}

First, all patients fulfilling the inclusion criteria were selected from the EAC. Then a symptom duration-matched cohort with Indian patients was selected from METEOR. Patients were matched 1:1 on symptom duration, to prevent that differences in symptom duration influenced the number of involved joints. Since fewer patients were available from Mexico and South Africa and average symptom duration was longer, these patients could not be matched 1:1. For these two countries, patients with the longest symptom duration were excluded to achieve sets of patients with similar symptom duration at baseline.

Frequencies were compared for autoantibody-positive (rheumatoid factor and/or ACPA positive) and autoantibody-negative (both rheumatoid factor and ACPA negative) patients and for patients from different countries. Comparisons between two groups were done with the Mann-Whitney $\mathrm{U}$ test, comparisons between several groups with the Kruskal-Wallis equality of populations rank test. $\mathrm{P}$ values $<0.05$ were considered statistically significant. All analyses were performed using Stata SE V.14 (StataCorp).

\section{RESULTS}

\section{Baseline characteristics}

Table 1 presents baseline characteristics. Patients in the Netherlands were more often autoantibody negative. Patients in Mexico and South Africa had a higher body mass index than Dutch and Indian patients. Since the number of autoantibody-negative patients in Mexico and South Africa was too low ( $\mathrm{n}=22$ and $\mathrm{n}=1$, respectively), analyses were only stratified for autoantibody status for the Netherlands and India. Baseline characteristics for 
Table 1 Comparison of baseline characteristics of patients with RA diagnosed in the Netherlands, India, Mexico and South Africa

\begin{tabular}{|c|c|c|c|c|c|c|c|c|}
\hline & \multicolumn{2}{|c|}{ Netherlands } & \multicolumn{2}{|c|}{ India } & \multicolumn{2}{|c|}{ Mexico* } & \multicolumn{2}{|c|}{ South Africa* } \\
\hline & $\mathbf{n}$ & $\begin{array}{l}\text { Mean } \\
\text { (SD) } / \%\end{array}$ & $\mathbf{n}$ & $\begin{array}{l}\text { Mean } \\
\text { (SD)/\% }\end{array}$ & $\mathbf{n}$ & $\begin{array}{l}\text { Mean } \\
\text { (SD) } / \%\end{array}$ & $\mathbf{n}$ & $\begin{array}{l}\text { Mean } \\
\text { (SD)/\% }\end{array}$ \\
\hline Gender (\% female) & 947 & 65.6 & 947 & 81.2 & 140 & 86.4 & 162 & 81.5 \\
\hline Age (years) & 947 & $56.8(15.3)$ & 942 & $42.9(12.6)$ & 131 & $47.7(12.5)$ & 163 & $47.7(12.5)$ \\
\hline RF (\% positive) & 942 & 61.4 & 944 & 87.3 & 137 & 80.3 & 164 & 99.4 \\
\hline ACPA (\% positive) & 886 & 51.5 & 442 & 72.4 & 99 & 60.6 & 91 & 96.7 \\
\hline Body mass index & 779 & $26.1(4.2)$ & 503 & $26.6(6.4)$ & 115 & $33.8(41.4)$ & 61 & $28.1(7.2)$ \\
\hline Cigarette smoking & 879 & & 908 & & 104 & & 151 & \\
\hline Current & & 24.0 & & 2.2 & & 24.0 & & 18.6 \\
\hline Past & & 36.6 & & 0.0 & & 3.9 & & 8.6 \\
\hline Never & & 39.4 & & 97.8 & & 72.1 & & 72.8 \\
\hline Symptom duration at diagnosis (months) & 947 & $5.3(4.7)$ & 947 & $5.8(4.5)$ & 141 & $5.3(2.5)$ & 154 & $5.4(2.4)$ \\
\hline DAS & 865 & $3.1(1.0)$ & 549 & $3.9(0.9)$ & - & - & - & - \\
\hline DAS28 & 867 & $5.0(1.4)$ & 517 & $6.1(1.3)$ & 124 & $5.6(1.3)$ & 137 & $5.9(1.3)$ \\
\hline ESR & 939 & $33.6(27.1)$ & 779 & $68.9(31.2)$ & 128 & 32.5 (19.3) & 141 & $39.6(28.7)$ \\
\hline CRP & 935 & $24.3(33.9)$ & 779 & $37.4(38.3)$ & 99 & $16.6(26.4)$ & 143 & $31.5(44.7)$ \\
\hline VAS patient global (mm) & 875 & $42.0(25.7)$ & 656 & $52.3(18.4)$ & 136 & $55.6(26.0)$ & 156 & $65.6(22.4)$ \\
\hline
\end{tabular}

*In order to achieve sets of patients with similar symptom duration at baseline, 86 patients from Mexico and 139 patients from South Africa with longest symptom duration were excluded.

ACPA, anti-citrullinated protein antibody; CRP, C-reactive protein; DAS, Disease Activity Score; ESR,

erythrocyte sedimentation rate; RA, rheumatoid arthritis; RF, rheumatoid factor; VAS, visual analogue scale.

autoantibody-positive and negative patients were similar, but autoantibody-positive patients had slightly longer symptom duration at diagnosis and autoantibody-negative patients in the Netherlands had higher disease activity (online supplementary file 1).

\section{Distribution of swollen and tender joints across countries}

The swollen joint distribution differed between countries (figure 1). In Dutch patients with RA, hand and foot joints were more often swollen than in India, especially MCP1-3, PIP2,3 and MTP2,3. In Mexico and South Africa, the distribution of swollen hand joints was similar to that in the Netherlands. In contrast to the Netherlands, knees were more often swollen in India, Mexico and South Africa ( $13 \%$ vs $30 \%, 37 \%$ and $36 \%$, respectively). Patients with RA in Mexico and South Africa also had more often swelling of other large joints than patients in India and the Netherlands. The shoulder was swollen in Mexico and South Africa in $21 \%$ and $11 \%$, compared with $0 \%$ and $1 \%$ in India and the Netherlands. Similarly for elbow joints these percentages were $29 \%, 23 \%, 7 \%$ and $7 \%$, respectively. In all countries, the number of tender joints was higher than the number of swollen joints, but patterns were similar.

When comparing the 28SJC between the four countries, the highest numbers of swollen joints were seen in Mexico and South Africa (median 7 and 8 vs 3 (India) and 5 (Netherlands)). Overall, tender joint count was highest in India (online supplementary file 2).

\section{Comparing autoantibody-positive and negative patients}

In general, the number of swollen and tender joints was higher in autoantibody-negative than in autoantibody-positive patients, especially in the Netherlands (online supplementary file 3). Despite higher joint counts, the swollen joint was very similar for autoantibody-positive and negative patients within both countries (figure 2).

\section{Sensitivity analyses}

Because of circularity between the number and distribution of swollen joints and fulfilment of the 2010 criteria, swollen joint distribution was also assessed in patients diagnosed with RA according to the rheumatologist. This showed similar distributions of swollen joints compared with patients fulfilling the 2010 criteria (online supplementary file 4 : figure 1 ).

\section{DISCUSSION}

This is the first large study comparing joint involvement in patients with recent-onset RA from different populations. In both the Netherlands and India, the distribution of involved joints did not differ for autoantibody-positive and negative patients within these countries, but joint involvement was reported differently in different countries. Synovitis in large joints was more frequent in India (knees), South Africa (knees, elbows) and Mexico (knees, elbows, shoulders) than in the Netherlands. Feet involvement was studied in two countries only and was considerably less frequently reported in India than in the Netherlands. 
A

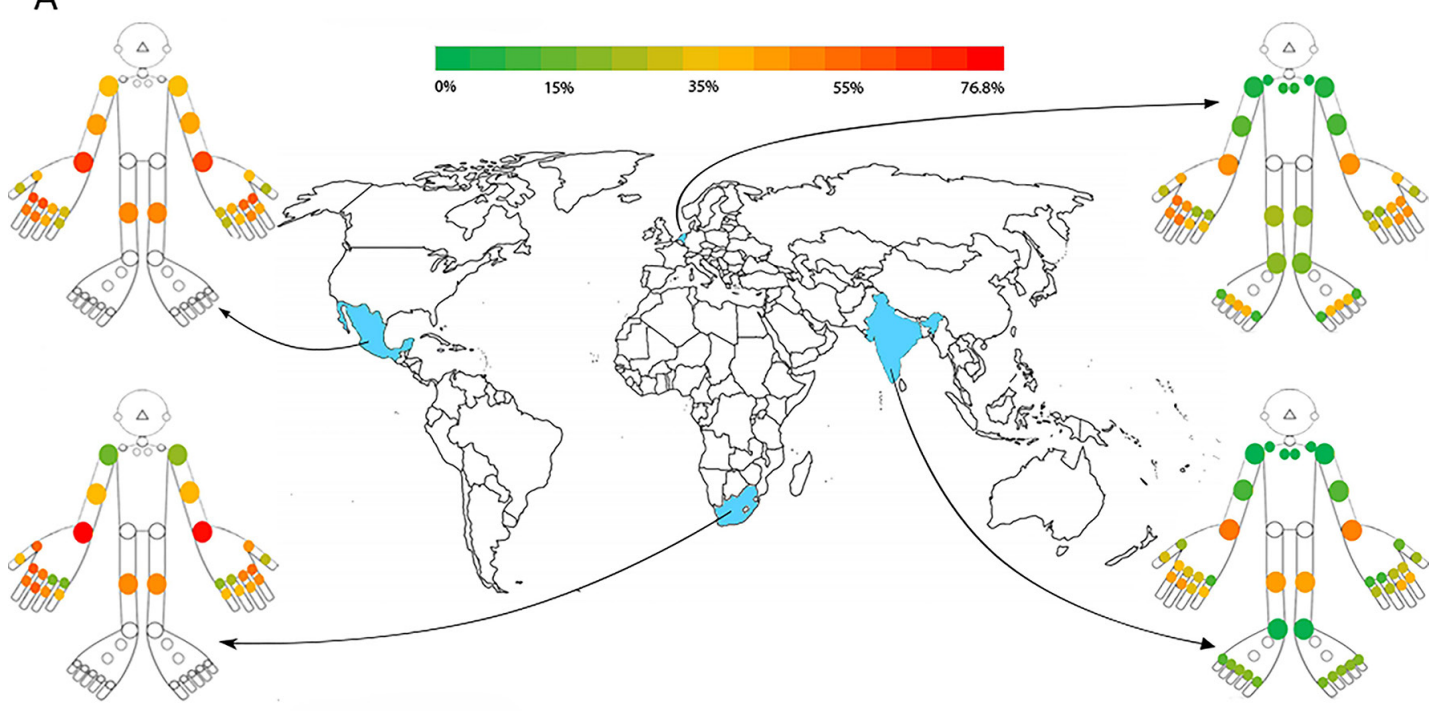

B

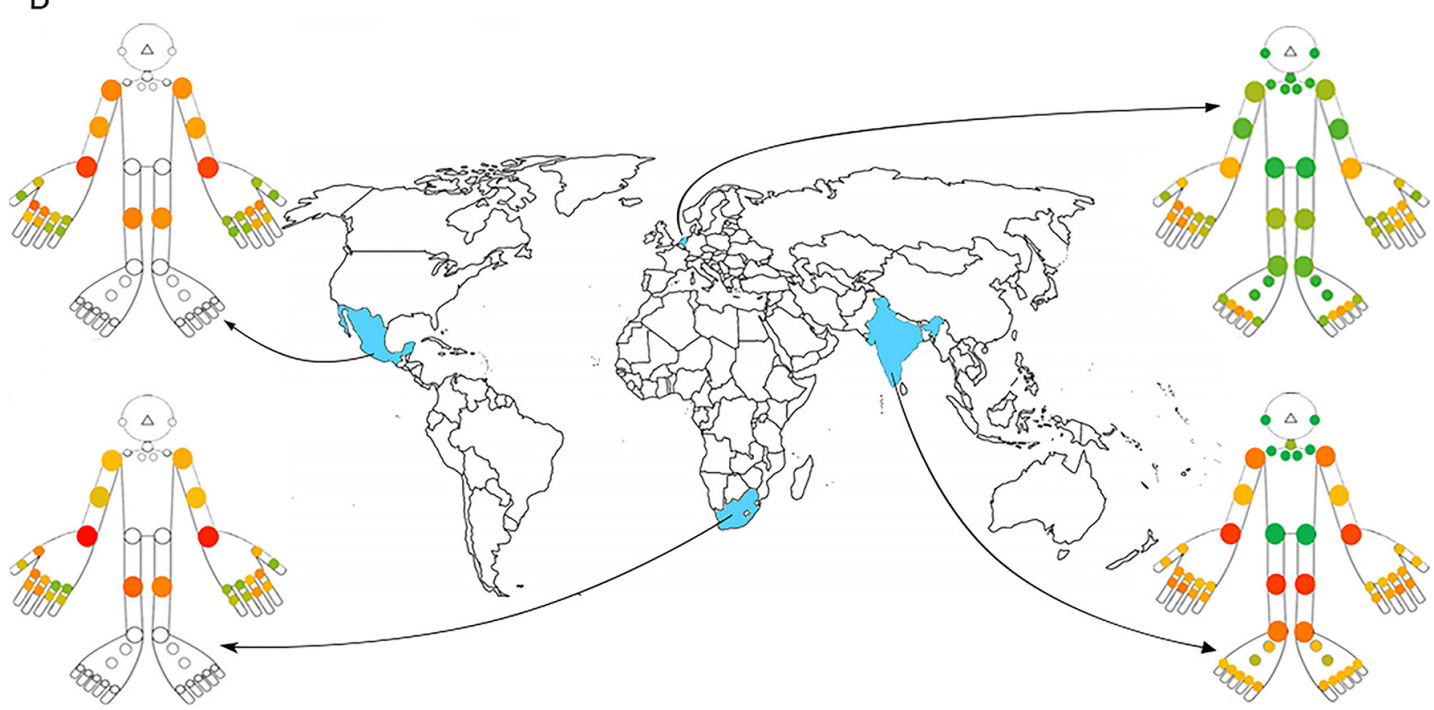

Figure 1 Distribution of swollen (-A) and tender (B) joint involvement in Mexico, the Netherlands, India and South Africa. Coloured circles indicate the percentage of patients with swelling or tenderness in the specific joint per country. White circles indicate that the joint is not included in the joint count.

Differences observed between the different countries can be caused by differences in reporting by doctors or in referral of patients to rheumatologists in the different countries. Then selection bias may have influenced the results. Alternatively, the observed differences can be 'true'. Then further studies on the underlying pathogenetic mechanisms that drive the difference in distribution of joint involvement are required and can increase our understanding on the mechanisms leading to RA and to inflammation of the predilection places. To evaluate which of these two possibilities is true, validation studies are required. This can either consist of validation studies in other observational cohorts, or based on analyses on randomised controlled trials. However, the inclusion criteria that are used in most intervention studies can also induce selection bias, as the patients studied here are often a severe subset of RA.

The consequences of different selection methods of patients between centres or countries, if present, do not affect the results of the analyses done within countries. Joint distribution of ACPA-positive and ACPA-negative RA has been studied previously and no differences were observed, which was now replicated in a larger Dutch data set as well as in an Indian RA population. ${ }^{7}$ This finding suggests that some common triggers lead to synovitis in similar joints in autoantibody-positive and negative patients, although these may differ between countries. Unfortunately, since the number of autoantibody-negative patients included in the database by a rheumatologists from Mexico and South Africa was limited, stratification on autoantibody positivity could not be performed for these data sets.

The number of autoantibody-positive patients was higher in India than in the Netherlands. This is intriguing as smoking is an important risk factor for ACPA-positive RA and the number of patients smoking in India was low. ${ }^{18}$ Different explanations are possible. It is known that the number of women smoking in India is low (approximately $3 \%) .{ }^{19}$ Another issue is that the smoking data captured contained information on cigarettes but not information 
A: Swollen Joint Involvement

Autoantibody negative

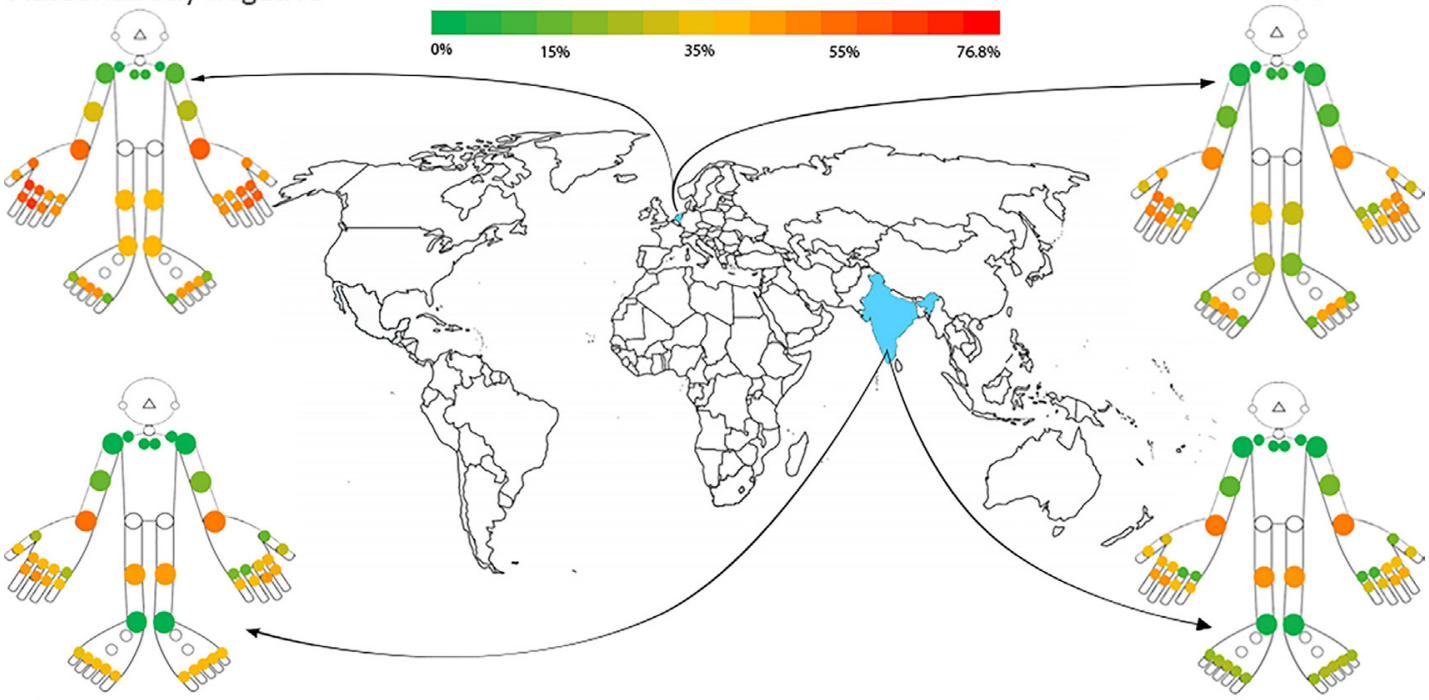

B: Tender Joint Involvement

Autoantibody negative

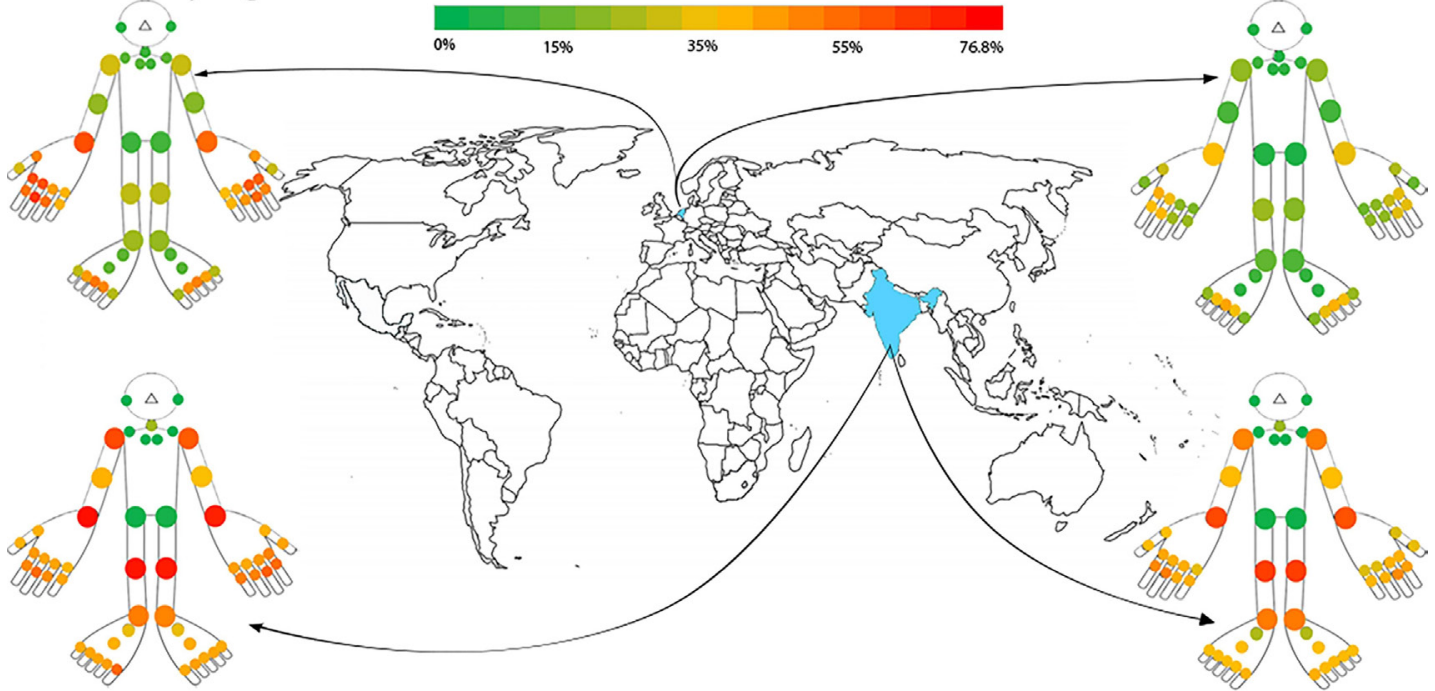

Autoantibody positive

(period between symptom onset and diagnosis). The date of start of symptoms as indicated by patients may be subject to recall bias. In addition, it is unknown if this is also subject to cultural differences.

Another limitation is that the data included in this study were captured during daily clinical practice and there has been no standardisation of joint counts between all contributing centres. Previous research showed that in particular, the interobserver reliability of swollen joint assessments is variable and studies on the effect of standardisation and training on swollen joint assessments show conflicting results. ${ }^{23}$ This could be a source of bias when comparing the distribution of involved joints between countries. However, in each centre multiple and different rheumatologists were involved as well, hence differences in joint examination between persons within at first presentation to rheumatologists in different countries, patients were matched on symptom duration 
and between centres may exist. The influence of such differences on the results is unknown. Another issue is that swelling of shoulder joints was prevalent in Mexico and South Africa. It is known that swelling is difficult to feel by palpation of this joint. To what extent the experienced rheumatologists also incorporated information on a reduced range of motion when filling information of joint counts is also unknown.

Patients were included when they were newly diagnosed and assessed the baseline visit. Therefore, rheumatologist-prescribed mediation was not yet started and it is highly likely that patients were disease-modifying antirheumatic drug naive, but we have no data on possible prescription of non-steroidal anti-inflammatory drugs or glucocorticoids by primary care physicians before the first visit to a rheumatologist. A final limitation is that all patients per country originated from a limited number of centres. Hence, we are unsure whether the patients included in different centres are reflective for the patient population of the entire country, especially for South Africa and Mexico for which included patient numbers were smaller.

In our view and despite all limitations, the present findings may be of interest for etiopathological studies. The vast majority of studies on genetic or environmental risk factors that are published now were done in Western countries. However, it is known that genetic risk factors differ around the world, for example, HLA-DRB1*09:01 is mainly associated with RA in Asian populations and African Americans but is not prevalent in Western populations, whereas PTPN22 is a risk factor specific to Caucasian populations. ${ }^{12}{ }^{24}$ To what extent different risk factors account for subtle differences in phenotypic presentation remains to be explored. However, such further studies may shed light on pathophysiological processes underlying the predilection locations of synovitis in early RA.

Contributors $\mathrm{SAB}$ analysed and interpreted the data and drafted the manuscript. AC, MS, DVM and NG were involved in data acquisition and manuscript revision. TWJH and AHM were involved in data acquisition, interpretation of data and manuscript revision. All authors read and approved the final manuscript.

Competing interests None declared.

Patient consent Obtained.

Ethics approval Ethical Committee of Leiden University.

Provenance and peer review Not commissioned; externally peer reviewed.

Data sharing statement The datasets used during the current study are available from the corresponding author on reasonable request.

Open Access This is an Open Access article distributed in accordance with the Creative Commons Attribution Non Commercial (CC BY-NC 4.0) license, which permits others to distribute, remix, adapt, build upon this work non-commercially, and license their derivative works on different terms, provided the original work is properly cited and the use is non-commercial. See: http://creativecommons.org/ licenses/by-nc/4.0/

(C) Article author(s) (or their employer(s) unless otherwise stated in the text of the article) 2017. All rights reserved. No commercial use is permitted unless otherwise expressly granted.
REFERENCES

1. Rantapää-Dahlqvist S, de Jong BA, Berglin E, et al. Antibodies against cyclic citrullinated peptide and IgA rheumatoid factor predict the development of rheumatoid arthritis. Arthritis Rheum 2003;48:2741-9.

2. Nielen MM, van Schaardenburg D, Reesink HW, et al. Specific autoantibodies precede the symptoms of rheumatoid arthritis: a study of serial measurements in blood donors. Arthritis Rheum 2004;50:380-6.

3. Song YW, Kang EH. Autoantibodies in rheumatoid arthritis: rheumatoid factors and anticitrullinated protein antibodies. QJM 2010;103:139-46.

4. Vossenaar ER, Zendman AJW, van Venrooij WJ. Citrullination, a possible functional link between susceptibility genes and rheumatoid arthritis. Arthritis Res Ther 2004;6:1-5.

5. Daha NA, Toes RE. Rheumatoid arthritis: Are ACPA-positive and ACPA-negative RA the same disease? Nat Rev Rheumatol 2011;7:202-3.

6. Derksen VF, Ajeganova S, Trouw LA, et al. Rheumatoid arthritis phenotype at presentation differs depending on the number of autoantibodies present. Ann Rheum Dis 2017;76:716-20.

7. van der Helm-van Mil AH, Verpoort KN, Breedveld FC, et al. Antibodies to citrullinated proteins and differences in clinical progression of rheumatoid arthritis. Arthritis Res Ther 2005;7:R949-58.

8. Ospelt C, Frank-Bertoncelj M. Why location matters - site-specific factors in rheumatic diseases. Nat Rev Rheumatol 2017;13:433-42.

9. Frank-Bertoncelj M, Trenkmann M, Klein K, et al. Epigeneticallydriven anatomical diversity of synovial fibroblasts guides jointspecific fibroblast functions. Nat Commun 2017;8:14852.

10. Stangenberg L, Burzyn D, Binstadt BA, et al. Denervation protects limbs from inflammatory arthritis via an impact on the microvasculature. Proc Natl Acad Sci U S A 2014:111:11419-24.

11. Ai R, Hammaker D, Boyle DL, et al. Joint-specific DNA methylation and transcriptome signatures in rheumatoid arthritis identify distinct pathogenic processes. Nat Commun 2016;7:11849.

12. Kochi Y, Suzuki A, Yamada R, et al. Ethnogenetic heterogeneity of rheumatoid arthritis-implications for pathogenesis. Nat Rev Rheumatol 2010;6:290-5.

13. Kanazawa T, Nishino J, Tohma S, et al. Analysis of the affected joints in rheumatoid arthritis patients in a large Japanese cohort. Mod Rheumatol 2013:23:44-9.

14. Peschken CA, Hitchon CA, Robinson DB, et al. Rheumatoid arthritis in a north american native population: Iongitudinal followup and comparison with a white population. J Rheumatol 2010;37:1589-95.

15. Aletaha D, Neogi T, Silman AJ, et al. rheumatoid arthritis classification criteria: an American College of Rheumatology/ European League Against Rheumatism collaborative initiative. Ann Rheum Dis 2010;2010:1580-8.

16. de Rooy DP, van der Linden MP, Knevel R, et al. Predicting arthritis outcomes--what can be learned from the Leiden Early Arthritis Clinic? Rheumatology 2011:50:93-100.

17. Bergstra SA, Machado PM, van den Berg R, et al. Ten years of METEOR (an international rheumatoid arthritis registry): development, research opportunities and future perspectives. Clin Exp Rheumatol 2016;34:S87-s90.

18. Linn-Rasker SP, van der Helm-van Mil AH, van Gaalen FA, et al. Smoking is a risk factor for anti-CCP antibodies only in rheumatoid arthritis patients who carry HLA-DRB1 shared epitope alleles. Ann Rheum Dis 2006;65:366-71.

19. World Lung Foundation. The tobacco atlas. $2015 \mathrm{http}: / / \mathrm{www}$. tobaccoatlas.org/country-data/india/ (accessed 17 Nov 2017).

20. Mishra GA, Pimple SA, Shastri SS. An overview of the tobacco problem in India. Indian J Med Paediatr Oncol 2012;33:139-45.

21. Linauskas A, de Thurah A, Andersen GN, et al. [No evidence that diet has any influence on the aetiology of rheumatoid arthritis]. Ugeskr Laeger 2015;177:V08140445.

22. de Smit MJ, Westra J, Brouwer E, et al. Periodontitis and rheumatoid arthritis: what do we know? J Periodontol 2015;86:1013-9.

23. Cheung PP, Gossec L, Mak A, et al. Reliability of joint count assessment in rheumatoid arthritis: a systematic literature review. Semin Arthritis Rheum 2014;43:721-9.

24. Reynolds RJ, Ahmed AF, Danila MI, et al. HLA-DRB1-associated rheumatoid arthritis risk at multiple levels in African Americans: hierarchical classification systems, amino acid positions, and residues. Arthritis Rheumatol 2014:66:3274-82. 